\title{
ON THE PROJECTIVE STRUCTURE OF A MODULAR LATTICE
}

\author{
R. M. THRALL
}

1. Introduction. The theory of projectivity ${ }^{1}$ has been considerably developed in the case of complemented modular lattices, and has been used in arbitrary modular lattices in connection with the JordanHölder theory and allied topics. The present note is devoted to the theory of projectivity in modular lattices with especial attention to the development of two canonical forms for projectivities. The first of these is given in $\$ 3$ (Theorem $A$ ) and the second in $\$ 4$. The main tool in the derivation of these forms is exploitation of the concept of projective root defined in $\$ 2$.

2. Definitions and notations. ${ }^{2}$ A quotient in a modular lattice $\mathfrak{R}$ is a pair of elements $x$ and $y$ in $\mathfrak{R}$ such that ${ }^{3} x \supset y$ and is denoted by the symbol $x / y$. Two quotients $a / b$ and $c / d$ are said to be transposes if either $a=b \cup c$ and $d=b \cap c$ or $c=a \cup d$ and $b=a \cap d$. Two quotients $a / b$ and $c / d$ are said to be projective if there exists a finite sequence $a / b, a_{1} / b_{1}, \cdots, c / d$ in which any two consecutive quotients are transposes. A quotient $a / b$ is called prime if there exists no element $c$ in $\&$ such that $a \supset c \supset b$.

Let $\mathfrak{R}$ be a modular lattice of finite dimension $l$ and let $\mathfrak{R}_{0}=\left\{0=v_{0} \subset v_{1} \subset \cdots \subset v_{l}=I\right\}$ be a maximal chain in $\mathfrak{R}$. Suppose that the $l$ prime quotients $v_{i-1} / v_{i}$ are separated into $r$ equivalence classes under the relation of projectivity, and let $l_{1}, \cdots, l_{r}$ be the orders of these classes. The partition $l=l_{1}+\cdots+l_{r}$ describes (although is far from characterizing) the projective structure in $\mathfrak{l}$, and is, in particular, independent of the choice of the chain $\Omega_{0}$. We call the unordered set of natural numbers $l_{1}, \ldots, l_{r}$ the projective structure constants (p.s.c.) of $\mathbb{R}$. A necessary and sufficient condition that $\mathbb{R}$ be distributive is that $r=l$. In the other extreme case $r=1, \mathbb{R}$ is said to be simple. ${ }^{4}$

Any $l$-dimensional sublattice $\mathfrak{R}^{\prime}$ of $\mathfrak{R}$ will have p.s.c. $l_{1}^{\prime}, \cdots, l_{r^{\prime}}^{\prime}$

\footnotetext{
Presented to the Society, September 2, 1949; received by the editors December 9, 1949.

${ }^{1}$ See Garrett Birkhoff, Lattice theory, rev. ed., Amer. Math. Soc. Colloquium Publications, vol. 25, New York, 1949, p. 72 and p. 118.

${ }^{2}$ Cf. Birkhoff, ibid., especially Chapter V. All of the concepts in this section are found in Birkhoff. The terminology "projective root" is new.

${ }^{3}$ Read " $x \supset y$ " as " $x$ properly contains $y . "$

4 Birkhoff, ibid., p. 78.
} 
with $r^{\prime} \geqq r$ such that the partition $l_{1}^{\prime}, \cdots, l_{r^{\prime}}^{\prime}$ is a refinement of the partition $l_{1}, \cdots, l_{r}$. (This follows from the fact that projectivity in a sublattice implies projectivity in the overlattice whereas the reverse implication is not true.)

One measure of the complexity of a modular lattice is how much it lacks being distributive. Each triple of elements in $\mathfrak{R}$ for which the distributive law does not hold generates a lattice which has a five element sublattice $\mathfrak{P}=\{r, s, t, u, w\}$ in which $r$ is the meet and $w$ is the join of each pair selected from $s, t, u$. We shall call such a five element lattice a projective root (p.r.) and shall use the notation $\mathfrak{P}=[r ; s, t, u ; w]$ to indicate that $\mathfrak{B}$ is a projective root. If any one (and therefore all) of the six quotients $s / r, t / r, u / r, w / s, w / t, w / u$ is prime in $\mathfrak{R}$, we say that $\mathfrak{B}$ is a prime projective root (p.p.r.) in $\mathfrak{R}$.

It is easily seen (we omit the proof) that if $\mathfrak{R}$ contains any projective root, then it contains a prime projective root. Indeed, if $\mathfrak{P}$ $=[r ; s, t, u ; w]$ is a p.r. with dimension $s / r=k$, then $\mathfrak{R}$ contains $k$ p.p.r.'s $\mathfrak{B}_{i}=\left[r_{i} ; s_{i}, t_{i}, u_{i} ; w_{i}\right], i=1, \cdots, k$, such that $r=r_{1}, w_{1}=r_{2}, \cdots$, $w_{k-1}=r_{k}, w_{k}=w$.

3. First normal form for a projectivity. So far as the definition of projectivity is concerned the successive quotients involved may wander all over the lattice according to no particular pattern. Our first step in the study of projectivities is, accordingly, the derivation of a normal form. This first normal form is without reference to the fixed chain $\mathfrak{R}_{0}$ and is valid for any modular lattice $\mathfrak{Z}$ in which all bounded chains are finite. In the next section a second normal form is set up which is adapted to the chain $\mathfrak{R}_{0}$ and in which almost all of the quotients used belong to a preassigned finite set which is defined in terms of $\Omega_{0}$.

Theorem A. Let $a / b$ be projective to $c / d$, both quotients being prime in $\mathbb{R}$. Then there exists $a$ sequence of transposes $a / b=x_{0} / y_{0}, x_{1} / y_{1}, \cdots$, $x_{k} / y_{k}=c / d$ such that

(i) No three consecutive $x_{i}$ (or $y_{i}$ ) form a chain.

(ii) For $k>2$ and for $i=1, \cdots, k-1$ either (a) $y_{i}=y_{i-1} \cap y_{i+1}$ or (b) $x_{i}=x_{i-1} \cup x_{i+1}$. Moreover, in case (a) we have $\mathfrak{B}_{i}=\left[y_{i} ; x_{i}, x_{i+1}\right.$ $\left.\cap y_{i-1}, x_{i-1} \cap y_{i+1} ; x_{i-1} \cap x_{i+1}\right]$ and in case (b) we have $\mathfrak{B}_{i}=\left[y_{i-1}\right.$ $\left.\cup_{y_{i+1}} ; y_{i}, y_{i-1} \cup_{x_{i+1}}, y_{i+1} \cup x_{i-1} ; x_{i}\right]$ as a prime projective root.

(iii) For $k=2$ there may or may not be a prime projective root related to the projectivity.

PRoof. If $x / x^{\prime}, y / y^{\prime}, z / z^{\prime}$ is a sequence of transposes for which $x \supset y \supset z$, then $x^{\prime} \cup_{z}=\left(x^{\prime} \cup y^{\prime}\right) \cup_{z}=x^{\prime} \cup\left(y^{\prime} \cup z\right)=x^{\prime} \cup y=x$. Similarly $x^{\prime} \cap z=z^{\prime}$. Hence, the middle quotient can be dropped from the se- 
quence and still leave a projectivity. By repeated applications of this process or dropping middle quotients we may assume that $a / b$ $=x_{0} / y_{0}, \cdots, x_{k} / y_{k}=c / d$ is a sequence of transposes which satisfies (i).

For each $i(0<i<k)$ we must have either $x_{i} \subseteq x_{i-1} \cap x_{i+1}$ or $x_{i} \supseteq x_{i-1}$ $\cup x_{i+1}$. The two possibilities are dual, so it is sufficient to treat only one, say the first. Then we have $x_{i} \cup y_{i+1}=x_{i+1}, x_{i} \cup y_{i-1}=x_{i-1}$, $x_{i} \cap y_{i-1}=x_{i} \cap y_{i+1}=y_{i}$. Let $x_{i}^{\prime}=x_{i-1} \cap x_{i+1}$ and let $y_{i}^{\prime}=y_{i-1} \cap y_{i+1}$. Then $x_{i} \cap y_{i}^{\prime}=x_{i} \cap y_{i-1} \cap y_{i+1}=y_{i}$, and $x_{i} \cup_{i}^{\prime}=x_{i} \cup\left(y_{i-1} \cap y_{i+1}\right) \subseteq x_{i}^{\prime}$. We consider two cases (A) $x_{i} \cup y_{i}^{\prime}=x_{i}^{\prime}$ and (B) $x_{i} \cup y_{i}^{\prime} \subset x_{i}^{\prime}$.

Case (A). Set $x_{i}^{\prime \prime}=x_{i-1} \cup x_{i+1}$ and $y_{i}^{\prime \prime}=y_{i-1} \cup y_{i+1}$. We shall show that the quotient $x_{i}^{\prime \prime} / y_{i}^{\prime \prime}$ is a transpose of both $x_{i-1} / y_{i-1}$ and $x_{i+1} / y_{i+1}$, and hence can be substituted for the quotient $x_{i} / y_{i}$ in the given sequence from $a / b$ to $c / d$. If $1<i<k-1$, we can then delete the quotients $x_{i-1} / y_{i-1}$ and $x_{i+1} / y_{i+1}$ from this altered sequence and have left a sequence of two less quotients connecting $a / b$ to $c / d$. If either $1<i$ or $i<k-1$, one of these deletions can still be made. At least one of these inequalities will always hold unless $1=i=k-1$, and therefore $k=2$, in which case there is nothing to be proved. The hypothesis of case (A) has as immediate consequence that $x_{i}^{\prime} / y_{i}^{\prime}$ is a transpose of both $x_{i-1} / y_{i-1}$ and $x_{i+1} / y_{i+1}$. Our desired conclusion for $x_{i}^{\prime \prime} / y_{i}^{\prime \prime}$ is accordingly a consequence of the following lemma.

Lemma A. Let $a / b$ and $c / d$ be prime quotients in a modular lattice ?. Then both of these quotients are transposes of $a \cap c / d \cap b$ if and only if they are both transposes of $a \cup c / b \cup d$.

Proof. "Only if." By hypothesis $a=b \cup(a \cap c)=a \cap(b \cup c)$ and hence $b \cup c=a \cup c$. Similarly, $a \cup d=a \cup c$. Moreover, $b \cap d=b \cap(a \cap c)$ $=b \cap c$, and similarly $b \cap d=a \cap d$. Now, $a \cap(b \cup d)=b \cup(a \cap d)$ $=b \cup(b \cap d)=b$, and $a \cup(b \cup d)=a \cup d=a \cup c$. Similarly $a \cup c / b \cup d$ is a transpose of $c / d$. The "if" part of the lemma follows from duality.

Case (B). We have $x_{i}^{\prime} \supset x_{i} \cup y_{i}^{\prime} \supset y_{i}^{\prime}$, and $\operatorname{so}{ }^{5} \operatorname{dim} x_{i}^{\prime} / y_{i}^{\prime} \geqq 2$. On the other hand $\operatorname{dim} x_{i}^{\prime} / y_{i}^{\prime} \leqq 2$ since $\operatorname{dim} x_{i-1} / y_{i-1}=\operatorname{dim} x_{i+1} / y_{i+1}=1$. Hence, $\operatorname{dim} x_{i}^{\prime} / y_{i}^{\prime}=2$ and the quotients $x_{i}^{\prime} / x_{i} \cup y_{i}^{\prime}$ and $x_{i} \cup y_{i}^{\prime} / y_{i}^{\prime}$ are both prime. From this it follows readily that $\left[y_{i}^{\prime} ; y_{i-1} \cap x_{i}^{\prime}, x_{i} \cup y_{i}^{\prime}\right.$, $\left.y_{i+1} \cap x_{i}^{\prime} ; x_{i}^{\prime}\right]$ is a p.p.r. Moreover, we can replace $x_{i} / y_{i}$ by $x_{i} \cup y_{i}^{\prime} / y_{i}^{\prime}$ in the given sequence of transposes and obtain a new sequence which still satisfies condition (i) and which satisfies condition (iia) at the $i$ th position.

The case where $x_{i} \supseteq x_{i-1} \cup_{i+1}$ is dual to the one just treated and leads, in case $k>2$, either to a shorter sequence of transposes or to a

${ }^{5}$ The dimension of a quotient $a / b$ is the dimension of the interval $[b, a]$; cf. Birkhoff, ibid., p. 11. 
sequence which satisfies condition (i) and which satisfies condition (iib) at the $i$ th position.

The theorem now follows immediately. The above proof also establishes the following corollary.

COROLLARY A. If $k$ is the shortest length of any sequence of transposes leading from $a / b$ to $c / d$, then there exists a sequence of transposes of length $k$ which satisfies all of the properties of the sequence of Theorem A.

4. The second normal form for a projectivity. The first normal form shows the importance of projective roots in the projective structure of a modular lattice. However, it sheds no direct light on the number of distinct prime projective roots that are necessary to set up projectivities between all pairs of prime quotients that are projective in a finite-dimensional modular lattice. We now describe a second normal form which is adapted to any maximal chain $\ell_{0}$ in $\&$; two preliminary concepts are needed.

Let $a / b$ be any prime quotient in $\mathbb{R}$ and consider the distributive sublattice $\mathfrak{D}^{\mathfrak{D}}$ of $\mathfrak{R}$ generated by $\mathfrak{R}_{0}$ and $a / b$. In $\mathfrak{D}$ the quotient $a / b$ will be projective to exactly one of the quotients $v_{i} / v_{i-1}, i=1, \cdots, l$. If this quotient is $v_{j} / v_{j-1}$, we say that $j=j(a / b)$ is the index of $a / b$ in $\mathfrak{R}$ relative to $\mathfrak{R}_{0}$. It may happen that $a / b$ is a transpose of $v_{j} / v_{j-1}$. If not, then either one of the quotients $v_{j} \cup a / v_{j-1} \cup b$ and $v_{j} \cap a / v_{j-1} \cap b$ can serve as the intermediate quotient to $a / b$ and $v_{j} / v_{j-1}$ in a sequence of transposes of length $k=2$, and any other sequence of transposes from $a / b$ to $v_{j} / v_{j-1}$ will have length greater than 2 .

To prove the above statements we consider the two chains

$$
\begin{aligned}
0 & =v_{0} \cap a \subseteq v_{1} \cap a \subseteq \cdots \subseteq v_{l} \cap a\left(=a=v_{0} \cup a\right) \\
& \subseteq v_{1} \cup a \subseteq \cdots \subseteq v_{l} \cup a=I, \\
0 & =v_{0} \cap b \subseteq v_{1} \cap b \subseteq \cdots \subseteq v_{l} \cap b\left(=b=v_{0} \cup b\right) \\
& \subseteq v_{1} \cup b \subseteq \cdots \subseteq v_{l} \cup b=I .
\end{aligned}
$$

In each of these chains there must be exactly $l$ proper inclusions. Since $\operatorname{dim} a / b=1$, there must be exactly one more proper inclusion in the subchain $v_{0} \cap a \subseteq \cdots \subseteq v_{l} \cap a$ than in the subchain $v_{0} \cap b \subseteq \cdots \subseteq v_{l}$ $\cap b$. Hence there is at least one index $j$ for which $v_{j} \cap a \supset v_{j-1} \cap a$ whereas $v_{j} \cap b=v_{j-1} \cap b$. Moreover, since $v_{j} \cap a \supset v_{j-1} \cap a \supseteq v_{j-1} \cap b$ $=v_{j} \cap b$ and $\operatorname{dim} v_{j} \cap a / v_{j} \cap b \leqq 1$ we conclude that $v_{j-1} \cap a=v_{j-1} \cap b$ $=v_{j} \cap b$. Now, $a / b, v_{j} \cap a / v_{j} \cap b\left(=v_{j} \cap a / v_{j-1} \cap a\right), v_{j} / v_{j-1}$ is a sequence

${ }^{B}$ Cf. Birkhoff, ibid., p. 27. 
of transposes which shows that $j$ is the index of the quotient $a / b$. The remaining statements follow from Lemma A.

The above argument also provides two new characterizations for the index $j(a / b)$. For we see that if $v_{j} \cap a \supset v_{j} \cap b=v_{j-1} \cap a$, then $j=j(a / b)$. Alternatively, $j(a / b)$ is the smallest index $j$ for which $v_{j} \cap a \supset v_{j} \cap b$.

We shall call a sequence of transposes from a prime quotient $a / b$ to $v_{j} / v_{j-1}$ where $j=j(a / b)$ a stem if it has minimal length $k$. According to the above paragraph we always have $k=1$ or $k=2$ for stems; if $k=1$ there is just one stem belonging to $a / b$ and if $k=2$ there are exactly two stems belonging to $a / b$. The reverse sequence of a stem is called a reverse stem.

A p.p.r. $\mathfrak{B}=[r ; s, t, u ; w]$ is said to be normal of type $i, j$ with respect to the chain $\Omega_{0}$ if $v_{i} / v_{i-1}, w / t, u / r, v_{j} / v_{j-1}$ is a sequence of transposes, and if $v_{i} \subseteq s \subseteq v_{j-1}$.

Lemma B. Let $a / b$ and $c / d$ be prime quotients in $\&$ with indices $j$ and $i$ respectively, and let $c=a \cup d, b=a \cap d$. Then $i \leqq j$, and if $i<j$ there

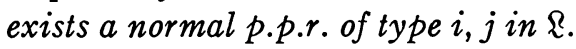

Proof. We have $v_{j} \cap c \supseteq v_{j} \cap d$. If equality holds, then $\left(v_{j} \cap d\right) \cap a$ $=\left(v_{j} \cap c\right) \cap a$ or $v_{j} \cap b=v_{j} \cap a$, which contradicts the hypothesis that $j=j(a / b)$. Hence, the inclusion is proper and so by the second alternative characterization of index we see that $j \geqq i=j(c / d)$.

Now suppose that $i<j$. Then the sequence of transposes $v_{j} / v_{j-1}$, $v_{j} \cap a / v_{j-1} \cap b, a / b, c / d, v_{i} \cup c / v_{i-1} \cup d, v_{i} / v_{i-1}$ can be shortened to one of length 3 by deletion of $a / b$ and $c / d$. Since $i<j$ and the $v_{h}$ form a chain, we cannot have any sequence of less than three transposes leading from $v_{j} / v_{j-1}$ to $v_{i} / v_{i-1}$. Hence, we can apply Theorem $\mathrm{A}$ and Corollary A to get a sequence of transposes $v_{j} / v_{j-1}=x_{0} / y_{0}, x_{1} / y_{1}$, $x_{2} / y_{2}, x_{3} / y_{3}=v_{i} / v_{i-1}$ such that $x_{2}=x_{1} \cup x_{3}, y_{1}=y_{0} \cap y_{2}$ and with the p.p.r.'s $\mathfrak{P}_{1}=\left[y_{1} ; x_{1}, y_{0} \cap x_{2}, x_{0} \cap y_{2} ; x_{0} \cap x_{2}\right]$ and $\mathfrak{P}_{2}=\left[y_{1} \cup y_{3} ; y_{2}, x_{1} \cup y_{3}\right.$, $\left.x_{3} \cup y_{1} ; x_{2}\right]$. (The fact that case (A) applies for $\mathfrak{B}_{1}$ and case (B) for $\mathfrak{P}_{2}$ follows from the form of the first sequence of length 3 obtained above connecting $v_{j} / v_{j-1}$ to $v_{i} / v_{i-1}$.)

Now, since $x_{0} \supseteq y_{0} \supset x_{3} \supset y_{3}$ we have $x_{0} \cap x_{2}=x_{0} \cap\left(x_{1} \cup x_{3}\right)=x_{1} \cup x_{3}$ $=x_{2}$, and similarly $y_{1} \cup y_{3}=y_{1}$. From these equalities we get $x_{0} \supseteq x_{2}$ $\supset y_{2}$ and $y_{3} \subseteq y_{1} \subset x_{1}$, and hence, $x_{0} \cap y_{2}=y_{2}$ and $x_{1} \cup y_{3}=x_{1}$. Finally, $x_{3} \cup y_{1}=x_{3} \cup\left(y_{0} \cap y_{2}\right)=\left(x_{3} \cup y_{2}\right) \cap y_{0}=x_{2} \cap y_{0}$. This shows that $\mathfrak{B}$ $=\left[y_{0} ; y_{1} \cup x_{3}, y_{2}, x_{1} ; x_{2}\right]$ is normal and of type $i, j$, which completes the proof of the lemma.

Let $\mathfrak{B}_{1}, \cdots, \mathfrak{P}_{m}$ (where $\mathfrak{P}_{h}=\left[r_{h} ; s_{h}, t_{h}, u_{h} ; w_{h}\right], h=1, \cdots, m$ ) be p.p.r.'s each normal with respect to $\mathfrak{R}_{0}$. A sequence of transposes 
$x_{0} / y_{0}, x_{1} / y_{1}, \cdots, x_{3 k} / y_{3 k}$ is said to be canonical with respect to $\mathfrak{P}_{1}, \cdots, \mathfrak{P}_{m}$ if each of the quotients $x_{0} / y_{0}, x_{3} / y_{3}, \cdots, x_{3 k} / y_{3 k}$ belongs to $\mathfrak{R}_{0}$, and if each pair $x_{3 s+1} / y_{3 s+1}, x_{3 s+2} / y_{3 s+2}, s=0, \cdots, k-1$ is of one of the forms $w_{h} / t_{h}, u_{h} / r_{h}$ or $u_{h} / r_{h}, w_{h} / t_{h}$ for suitable $h=h(s)$, and if no quotient appears twice in the sequence.

Lemma C. There exist $l-r$ distinct prime projective roots $\mathfrak{B}_{h}$ $=\left[r_{h} ; s_{h}, t_{h}, u_{h} ; w_{h}\right], h=1, \cdots, l-r$, each normal with respect to $\mathfrak{R}_{0}$, and such that the sublattice $\mathfrak{R}^{\prime}$ of $\mathfrak{R}$ generated by $\mathfrak{R}_{0}$ and $\mathfrak{P}_{1}, \cdots, \mathfrak{P}_{l-r}$ has the same projective structure constants as $\mathfrak{R}$. Moreover, if $v_{i} / v_{i-1}$ is

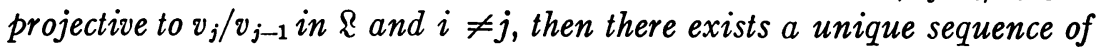
transposes canonical with respect to $\mathfrak{B}_{1}, \cdots, \mathfrak{B}_{l-r}$, which establishes this projectivity.

Proof. If $\mathfrak{R}$ is distributive, there is nothing to prove. If $\mathfrak{R}$ is not distributive, then we have at least two distinct quotients $v_{i} / v_{i-1}$ and $v_{j} / v_{j-1}$ which are projective in $\&$. Let $v_{i} / v_{i-1}=x_{0} / y_{0}, x_{1} / y_{1}, \cdots, x_{k} / y_{k}$ $=v_{j} / v_{j-1}$ be any sequence of transposes which establishes this projectivity. Let $x_{h} / y_{h}$ be the first quotient in this sequence whose index is not equal to $i$. Then, according to Lemma $\mathrm{B}, \mathbb{R}$ contains a normal p.p.r. $\mathfrak{B}_{1}$ of type $i_{1}, j_{1}$ where $i_{1}=\operatorname{minimum}\left(i, j\left(x_{h} / y_{h}\right)\right)$ and $j_{1}=$ maxi$\operatorname{mum}\left(i, j\left(x_{h} / y_{h}\right)\right)$.

We now take as an induction hypothesis that for some $h<l-r$ we have found $h$ p.p.r.'s $\mathfrak{B}_{1}, \cdots, \mathfrak{B}_{h}$ with $\mathfrak{B}_{m}$ normal of type $i_{m}, j_{m}$, $m=1, \cdots, h$, such that each of the graphs $G_{m}$ described below is a forest (that is, contains no cycle). Each graph $G_{m}$ has the $l$ vertices $Q_{1}, \cdots, Q_{l}$, and we say that $Q_{i}$ is associated with the quotient $v_{i} / v_{i-1}$. In $G_{0}$ there are no edges, and $G_{m}$ (for $m>0$ ) has the $m$ edges $\left(Q_{i_{1}}, Q_{j_{1}}\right), \cdots,\left(Q_{i_{m}}, Q_{j_{m}}\right)$. Clearly, $G_{h}$ has exactly $l-h$ connected subgraphs. Now, since $l-h>r$, there must be some pair of quotients

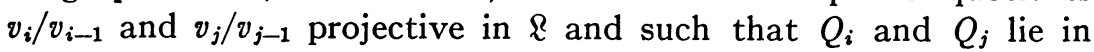
distinct connected subgraphs of $G_{h}$. Let $v_{i} / v_{i-1}=x_{0} / y_{0}, x_{1} / y_{1}, \cdots$, $x_{k} / y_{k}=v_{j} / v_{j-1}$ be any sequence of transposes establishing the projectivity of the given quotients. Then, since the indices of $x_{0} / y_{0}$ and of $x_{k} / y_{k}$ give corresponding vertices $Q_{i}$ and $Q_{j}$ belonging to distinct connected subgraphs of $G_{h}$, there must be some consecutive pair of quotients in the given sequence with indices $i^{\prime}$ and $j^{\prime}$ such that $Q_{i^{\prime}}$ and $Q_{j}$ lie in distinct connected subgraphs of $G_{h}$. Now apply Lemma $\mathrm{B}$, and we get a p.p.r. $\mathfrak{P}_{h+1}$ of type $i_{h+1}, j_{h+1}$ where $i_{h+1}$ is the minimum and $j_{h+1}$ is the maximum of the two integers $i^{\prime}$ and $j^{\prime}$.

Completing this induction argument we arrive at the following position. We have $l-r$ p.p.r.'s $\mathfrak{B}_{1}, \cdots, \mathfrak{B}_{l-r}$ and a corresponding graph $G_{l \rightarrow r} \cdot G_{l-r}$ is a forest with exactly $r$ connected subgraphs. Moreover, 
two quotients $v_{i} / v_{i-1}$ and $v_{j} / v_{j-1}$ are projective in $\mathfrak{R}$ if and only if the corresponding vertices $Q_{i}$ and $Q_{j}$ belong to a connected subgraph of $G_{l-r}$; hence, the sublattice $\mathfrak{R}^{\prime}$ of $\mathfrak{R}$ generated by $\mathfrak{R}_{0}$ and the $\mathfrak{B}_{h}$ has the same p.s.c. as $\mathbb{R}$. With the directed edge $\left(Q_{i_{h}}, Q_{j_{h}}\right)$ of the graph we associate the sequence of transposes $v_{i_{h}} / v_{i_{h}-1}, w_{h} / t_{h}, u_{h} / t_{h}, v_{j_{h}} / v_{j_{h}-1}$. We associate the reverse sequence with the same edge oppositely directed. By fitting such sequences together we associate with any connected path in $G_{l-r}$ without any repeated vertex and leading from a vertex $Q_{i}$ to a vertex $Q_{j}$ a sequence of transposes canonical with respect to $\mathfrak{P}_{1}, \cdots, \mathfrak{P}_{l-r}$ and leading from $v_{i} / v_{i-1}$ to $v_{j} / v_{j-1}$. Conversely, with any canonical sequence of transposes we may associate a connected path in $G_{l-r}$ leading from $Q_{i}$ to $Q_{j}$. Since the graph $G_{l-r}$ is a forest, there is at most one connected path leading from $Q_{i}$ to $Q_{j}$ provided no vertex is used twice; this establishes the uniqueness part of the lemma, and completes the proof.

As a consequence of Lemma $\mathrm{C}$ we see that if two prime quotients $a / b$ and $c / d$ are projective in $\mathbb{R}$, there exists a sequence of the form stem-canonical sequence-reverse stem which establishes this projectivity. The canonical sequence used is uniquely defined by the given quotients (more precisely by the indices of $a / b$ and $c / d$ ), and for each of the stem and of the reverse stem there are at most two choices. A sequence of the form stem-canonical sequence-reverse stem is said to be in the second normal form for a projectivity.

As compared with the first normal form the second one may be much longer and need not satisfy the conditions of the first normal form. However, in the second form there is an economy in the total number of prime projective roots used, and it is essentially uniquely determined by its initial and terminal quotients. The second normal form is expected to be of especial importance in the study of sublattices of the lattice of all subspaces of a finite-dimensional vector space.

UNIVERSITY OF MichigaN 